\title{
ABC of chronic obstructive pulmonary disease Non-pharmacological management
}

Graeme P Currie, J Graham Douglas

Chronic obstructive pulmonary disease (COPD) is a progressive and largely irreversible disorder. Unsurprisingly, drugs alone cannot ensure optimum short and long term outcomes. As a consequence, there is increasing interest in the role of non-drug strategies and multi-disciplinary team input in the overall management of COPD.

\section{Pulmonary rehabilitation}

Depending on local availability, consider referring all COPD patients-irrespective of age, functional limitation, and smoking status-for pulmonary rehabilitation. This is "a multidisciplinary programme of care for patients with chronic respiratory impairment that is individually tailored and designed to optimise physical and social performance and autonomy." A suitable programme is important in breaking the vicious cycle of worsening breathlessness, reduced physical activity, and deconditioning that many patients experience, and pulmonary rehabilitation plays a major role in restoring patients to an optimally functioning state. For example, early intervention after an acute exacerbation of COPD can produce clinically significant improvements in exercise capacity and health. The ideal programme should consist of several components, including exercise training, education, and nutritional support.

Exercise training-Outpatient pulmonary rehabilitation programmes typically run for two months, with two or three sessions of supervised exercise each week. Patients are encouraged to exercise at home and record their achievements, so that progress can be monitored. Studies have shown that physiological changes provided by endurance training take place at the level of skeletal muscle, even during sub-maximal exercise. Exercise training can improve exercise tolerance, symptoms, quality of life, peak oxygen uptake, endurance time during sub-maximal exercise, functional walking distance, and peripheral and respiratory muscle strength.

Education-This generally comprises various forms of goal directed and systematically applied communication aimed at improving understanding and motivation. The programme should be structured, and potential topics include breathing control, relaxation, benefits of exercise, and value of smoking cessation. Although education individualised to the patient is often helpful, group based education may be more effective. Participants are encouraged to take responsibility for their own health, and follow-up sessions may be necessary at home.

Nutritional advice-The term COPD encompasses patients previously labelled as being overweight "blue bloaters" and underweight "pink puffers." Despite the marked differences in body habitus and nutritional status, the pathophysiological hallmark in both phenotypes is relatively fixed airflow obstruction. Many COPD patients are underweight because of the heightened energy output associated with the increased work of breathing and reduced nutritional intake due to limitations posed by severe breathlessness. However, patients can become overweight because of reduced activity and overeating. The reasons for these phenotypic differences are far from clear, but raised concentrations of cytokines such as tumour necrosis factor $\alpha$ and leptin have been implicated in weight loss.

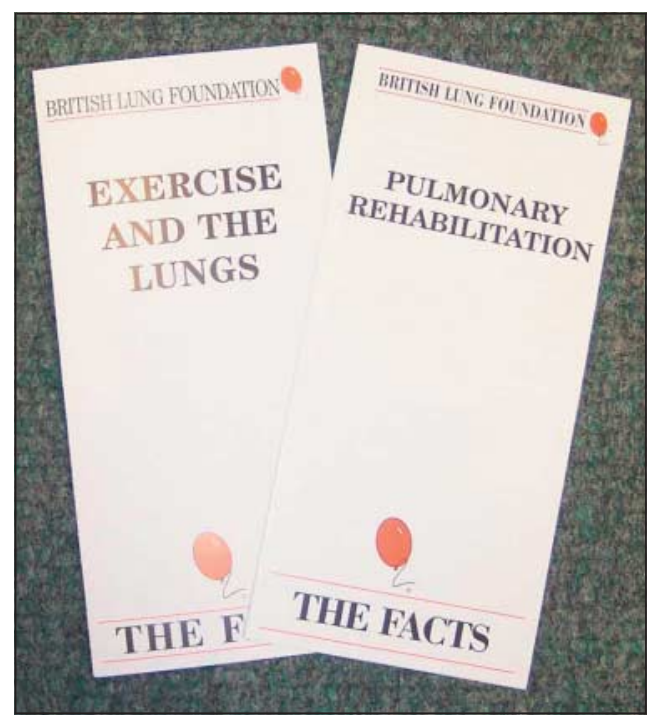

Pulmonary rehabilitation should be offered to most patients with COPD

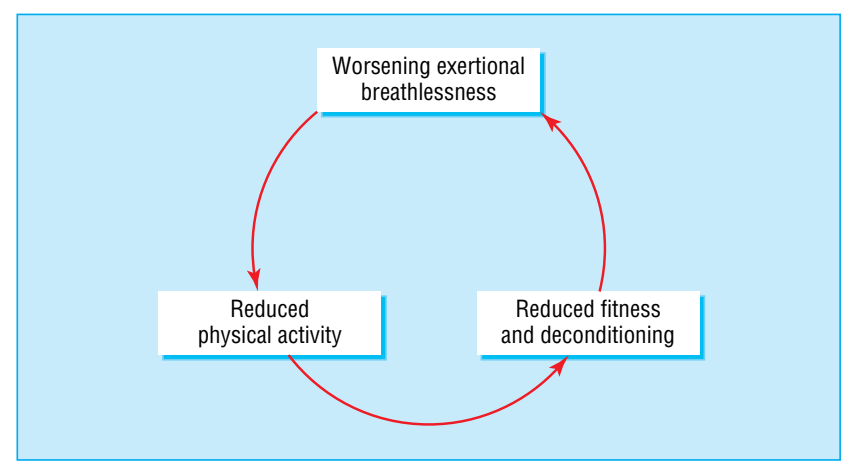

A determined attempt should be made to break the vicious circle of worsening breathlessness, reduced physical activity, and deconditioning 


\section{Long term outcomes of pulmonary rehabilitation} Improved exercise performance and reduced breathlessness associated with exercise can be maintained for up to 12 months. Pulmonary rehabilitation can also improve quality of life, but this benefit tends to decline over time. Moreover, there is no influence on disease progression (as judged by decline in forced expiratory volume in 1 second $\left(\mathrm{FEV}_{1}\right)$ ), and there is little evidence of improvement in long term survival. Data about use of healthcare resources are conflicting, with no consistent reduction in hospital admission rates or length of hospital stay.

\section{Immunisation}

Many exacerbations of COPD are caused by viral and bacterial infection, suggesting that vaccination could reduce the number of exacerbations. Unless they are contraindicated, arrange pneumococcal and influenza immunisation in all patients with COPD.

Pneumococcal vaccination-Current pneumococcal vaccines contain $25 \mathrm{ng}$ of purified capsular polysaccharide from each of 23 subtypes of Streptococcus pneumoniae. A single dose of $0.5 \mathrm{ml}$ is given intramuscularly, and mild soreness and induration at the site of injection is common. Re-immunisation is not advised and is contraindicated within three years. The severe reactions to re-immunisations that occur are probably due to high levels of circulating antibodies. Although evidence suggests that the current 23 valent vaccine is effective in preventing the bacteraemia associated with pneumococcal pneumonia, there is no convincing evidence of benefit to patients with COPD.

Influenza vaccination-Influenza vaccine is prepared each year with viruses (usually two type A and one type $\mathrm{B}$ ) similar to those considered most likely to be circulating in the forthcoming winter. The viruses are grown in the allantoic cavity of chick embryos, and the vaccine is therefore contraindicated in people with egg allergy. Patients are often concerned about adverse effects, and doubts may exist about the protective effects of the vaccine. A Cochrane review of 20 cohort studies evaluating the effects of inactivated influenza vaccine in high risk patients (including some with COPD) confirmed that vaccination did confer an overall reduction in exacerbation rates. In another meta-analysis of patients given influenza vaccination, the pooled estimates of efficacy for patients older than 65 years were $56 \%$ for preventing respiratory illness, $53 \%$ for preventing pneumonia, $50 \%$ for preventing hospital admission, and $68 \%$ for preventing death.

\section{Mental health status}

Many patients with COPD have anxiety and depression, which are likely to further impair quality of life. These conditions are probably multifactorial in origin, with social isolation, persistent symptoms, and inability to participate in many activities of daily living all likely to play a role. Positively search for features suggestive of an anxiety or depressive disorder. Mental health status can be assessed with simple tools such as the hospital anxiety and depression scale. If anxiety or depression is present, treat with conventional drugs, although care should be taken with the use of benzodiazepines and other respiratory depressants. Common sense also suggests that an aggressive attempt to optimise lung function and improve substantial hypoxaemia should be undertaken in these patients.

\section{Surgery}

Many COPD patients are unsuitable for surgical intervention because of high operative risk, and careful consideration is
Overall aims of treatment of COPD

- Reduce symptoms

- Improve exercise tolerance

- Improve health related quality of life

- Prevent exacerbations

- Provide package of care that meets the patient's needs

- Provide treatment that minimises the risk of adverse effects

- Reduce mortality

- Prevent disease progression

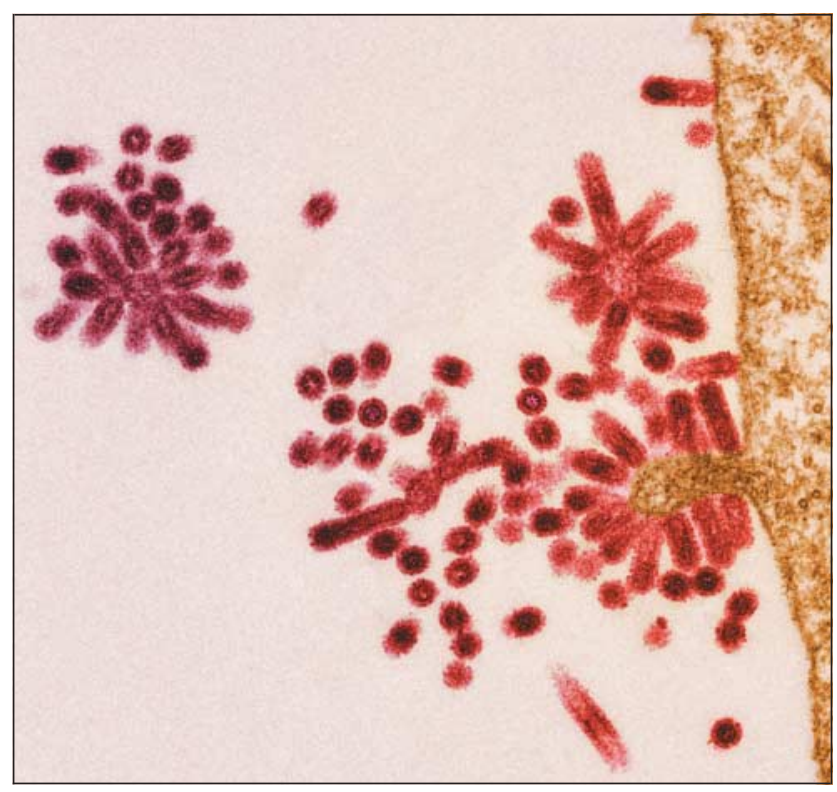

Electron micrograph showing influenza viruses (red) budding from a host cell

\section{Hospital anxiety and depression scale}

Scoring is based on a 4 point scale (0-3). A score of 0-7 is normal, 8-10 borderline, and 11-21 suggests moderate to severe anxiety or depression

Anxiety

- I feel tense or wound up (Most of the time (3) to Not at all (0))

- I get sort of frightened feelings as if something awful is going to happen (Definitely and badly (3) to Not at all (0))

- Worrying thoughts go through my mind (Definitely (3) to Not at all (0))

- I can sit at ease and feel relaxed (Definitely (0) to Not at all (3))

- I get a sort of frightened feeling like butterflies in the stomach (Not at all (0) to Very often (3))

- I feel restless as if I have to be on the move (Very much (3) to Not at all (0))

- I get sudden feelings of panic (Very often (3) to Not at all (0))

Depression

- I look forward with enjoyment to things (As much as I ever did (0) to Hardly at all (3))

- I have lost interest in my appearance (Definitely (3) to I take as much care as ever (0))

- I still enjoy the things I used to enjoy (Definitely as much (0) to Hardly at all (3))

- I can laugh and see the funny side of things (As much as I always could (0) to Not at all (3))

- I feel cheerful (Not at all (3) to Most of the time (0))

- I feel as if I am slowed down (Nearly all the time (3) to Not at all (0),

- I can enjoy a good book, the radio, or a TV programme (Often (0) to Seldom (3)) 
required before contemplating a procedure. The three most common procedures-bullectomy, lung volume reduction surgery, and transplantation-carry substantial short and long term risks of morbidity and mortality. They are therefore generally limited to motivated former smokers who have serious symptoms despite maximal treatment.

Bullectomy-In some patients with COPD, bullae can occupy large volumes of the thoracic cavity, causing compression of surrounding functional lung parenchyma. Bullectomy should be considered in symptomatic patients with a large bulla-especially those with moderate or severe airflow obstruction, a prior pneumothorax, or haemoptysis. Differentiating a pneumothorax from a large lung bulla can be difficult, and computed tomography of the chest may be required. Inadvertent insertion of a chest drain into a bulla can lead to complications such as the development of a bronchopleural fistula.

Lung volume reduction surgery-Lung volume reduction surgery involves removal of segments of inefficient emphysematous lung parenchyma in order to promote better gas exchange in the remaining, less affected part. The procedure can improve quality of life, exercise capacity, and lung function in carefully selected individuals, and, in subgroup analysis in some studies, prolong survival. Moreover, it is a safer procedure than lung transplantation and avoids the problem of lack of donor lungs. Consider surgical referral of patients fulfilling the criteria of predominantly upper lobe disease, markedly impaired exercise capacity despite appropriate treatment, and $\mathrm{FEV}_{1}>20 \%$ of the predicted value. Recently, interest has been growing in bronchoscopic lung volume reduction in patients with COPD. This involves obstructing emphysematous areas of lung with, for example, an endobronchial valve--therefore avoiding the risks associated with major surgery. The potential role of this procedure in treating COPD requires further evaluation.

Lung transplantation-Some motivated former smokers with COPD should be considered for lung transplantation, although, as in most transplant procedures, this is greatly limited by organ availability. Patients with concomitant medical conditions and advanced age generally have poor survival rates. The upper age limit is generally considered to be 60 years for a bilateral lung transplant, and 65 years for a single lung transplant. Local guidelines should be consulted, but suggested criteria for referral include patients with a combination of advanced airflow obstruction, significant functional impairment, cor pulmonale, and life expectancy of less than two years.

\section{Multidisciplinary care}

Members of a multidisciplinary team can greatly assist patients with the physical, domestic, and social limitations imposed by severe breathlessness. Respiratory nurse specialists are likely to have an increasingly important role and can provide a vital interface between primary and secondary care. Areas where they can provide support include helping patients deal with emotional and psychological sequelae, patient education, assessing inhaler technique, nebuliser assessments, nurse prescribing, organisation of assisted discharge schemes, monitoring domiciliary non-invasive ventilation, and end of life palliation and family support.

Graeme P Currie is specialist registrar and J Graham Douglas is consultant in the Respiratory Unit, Aberdeen Royal Infirmary, Aberdeen.

The $\mathrm{ABC}$ of chronic obstructive pulmonary disease is edited by Graeme P Currie. The series will be published as a book by Blackwell Publishing in autumn 2006.

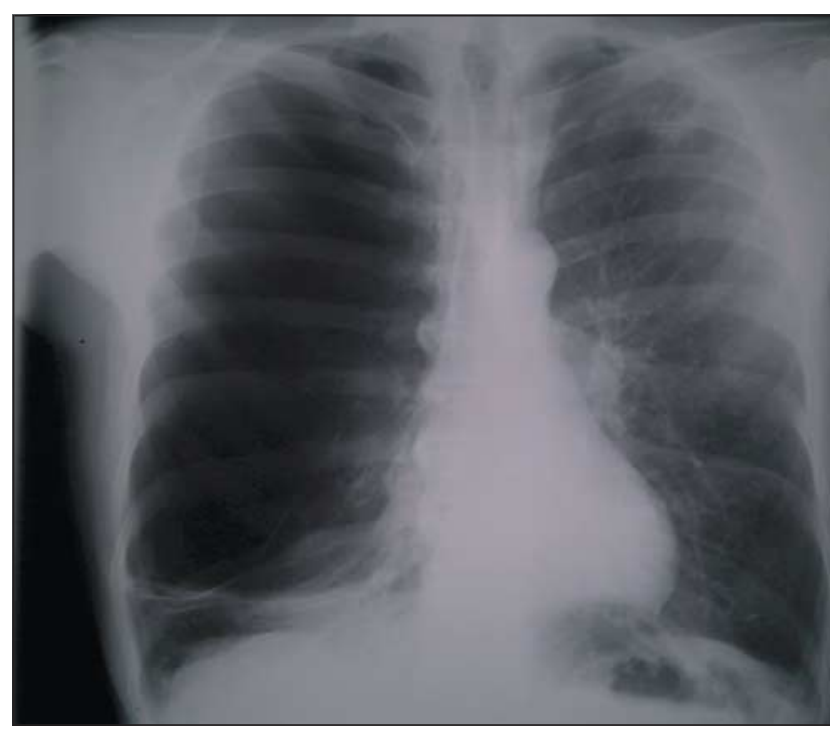

A large right sided lung bulla in a patient with COPD

\section{Multidisciplinary team members often}

required for optimal care of patients with COPD

- Hospital doctors

- General practitioners

- Respiratory nurse specialists

- Social workers

- Dietitians

- Occupational therapists

- Pharmacists

- Mental health care workers

- Physiotherapists

\section{Further reading}

- British Thoracic Society guidelines. Pulmonary rehabilitation. Thorax 2001;56:827-34

- Man WD, Polkey MI, Donaldson N, Gray BJ, Moxham J. Community pulmonary rehabilitation after hospitalisation for acute exacerbations of chronic obstructive pulmonary disease: randomised controlled study. BMJ 2004;329:1209-11

- Gross PA, Hermogenes AW, Sacks HS, Lau J, Levandowski RA. The efficacy of influenza vaccine in elderly persons. A meta-analysis and review of the literature. Ann Intern Med 1995;123:518-27

- Poole PJ, Chacko E, Wood-Baker RWB, Cates CJ. Influenza vaccine for patients with chronic obstructive pulmonary disease. Cochrane Database Syst Rev 2000;(4):CD002733

- Ferreira IM, Brooks D, Lacasse Y, Goldstein RS. Nutritional support for individuals with COPD: a meta-analysis. Chest 2000;117:672-8

- Fishman A, Martinez F, Naunheim K, Piantadosi S, Wise R, Ries A, et al. A randomized trial comparing lung-volume-reduction surgery with medical therapy for severe emphysema. $N$ Engl J Med 2003;348:2059-73

- Hopkinson NS, Toma TP, Hansell DM, Goldstraw P, Moxham J, Geddes DM, et al. Effect of bronchoscopic lung volume reduction on dynamic hyperinflation and exercise in emphysema. Am J Respir Crit Care Med 2005:171:453-60

Competing interests: GPC has received funding for attending international conferences and honoraria for giving talks from pharmaceutical companies GlaxoSmithKline, Pfizer, and AstraZeneca. JGD has received funding for attending international conferences from GlaxoSmithKline and Novartis; fees for speaking from Boehringer, AstraZeneca, Atlanta, and GlaxoSmithKline; and research funding from Boehringer and GlaxoSmithKline.

The electron micrograph of influenza virus is reproduced with permission of Steve Gschmeissner and Science Photo Library.

BMJ 2006;332:1379-81 